http://jmscr.igmpublication.org/home/

ISSN (e)-2347-176x ISSN (p) 2455-0450

crossref DOI: https://dx.doi.org/10.18535/jmscr/v7i8.111

Journal Of Medical Science And Clinical Research

\title{
A Study on the Evaluation of CBNAAT -A Novel Diagnostic tool for Rapid and Specific Diagnosis of Mycobacterium Tuberculosis in Pulmonary and Extrapulmonary Specimens in a Tertiary Care Hospital of South India
}

Authors

\author{
Dr Muthukumar Shunmugam ${ }^{1}$, Dr Joseph Pratheeban², Dr Karthiga ${ }^{3}$, Dr Gayathri ${ }^{4}$ \\ ${ }^{1}$ Associate Professor, Department of Pulmonary Medicine, Kanyakumari Government Medical College \\ Hospital \\ ${ }^{2,3}$ Assistant Professor, Department of Pulmonary Medicine, Kanyakumari Government Medical College \\ Hospital \\ ${ }^{4}$ Post Graduate, Department of General Medicine, Kanyakumari Government Medical College Hospital
}

\begin{abstract}
Background: Among the infectious diseases Tuberculosis $(T B)$ is one of the most dangerous threats to public health. As per the Global TB report 2017 the estimated incidence of TB in India was approximately 28,00,000 accounting for about a quarter of the world's TB cases. We need accurate feasible, rapid and affordable diagnostic tool to detect Mycobacterium Tuberculosis .Among various investigations in diagnosis of TB, Genexpert which was recently endorsed by WHO remains one of the rapid method to detect Mycobacterium Tuberculosis in both pulmonary and extra pulmonary specimens. Recently, there has been increasing evidence that supports the use of this assay in clinical samples other than sputum for patients with Extra Pulmonary TB.

Materials and Methods: This is a retrospective observational study conducted in Department of Pulmonary medicine in Kanyakumari government medical college hospital. Pulmonary and extra pulmonary specimens taken from the patients which included outpatients and inpatients attending the tertiary care centre with TB suspect .Around 6638 samples taken from July 2017 to 2018 December were included in the study.Both pulmonary and extra pulmonary samples were taken for the study after informed consent. Gene Xpert System is equipped with GX2.1 software; computer; printer; barcode ; wand-reader and operator manual (Cepheid Inc, Sunnyvale, USA), this is available in a one, two, four, or 16-module configuration The Gene Xpert Cartridge is a single-use disposable cartridge all the processes including Sample extraction, amplification and detection are all carried out within this self-contained cartridge. The sputum sample collected from the patient with suspected TB is mixed with the reagent that is provided with the assay, and a cartridge containing this mixture is placed in the GeneXpert machine further processing from this point is fully automated.

Results: About 6638 samples have been taken from July 2017 to 2018 December.Out of this 1152 samples detected Mycobacterium tuberculosis. Out of this 1111 were sensitive to rifampicin and 41 were resistant to rifampicin. 5486 samples did not detect Mycobacterium tuberculosis. 41 cases where positive for HIV .1073 samples were pulmonary while 79 samples were extrapulmonary which included specimens from CSF, Pleural fluid \& Ascitic fluid .

Conclusion: From this study We concluded that the MTB/RIF test is a simple method to detect MTB. In conclusion, this assay not only has better sensitivity and specificity for the diagnosis of TB and detection of rifampin resistance in few hours, but also requires minimal biosafety measures. However, the new molecular technique is considerably more expensive, because of the high price of reagents and instrument maintenance, than conventional methods. Still this assay has a high clinical application value in earlier diagnosis of both pulmonary and extra pulmonary TB which can be applied for the early diagnosis and treatment of the disease.

Keywords: $A F B, C B N A A T, M T B, R I F$.
\end{abstract}




\section{Introduction}

Among the infectious diseases Tuberculosis (TB) is one of the most dangerous threats to public health. As per the Global TB report 2017 the estimated incidence of TB in India was approximately 28,00,000 accounting for about a quarter of the world's TB cases. GeneXpert MTB/RIF assay is a nucleic acid amplification (NAA) test which simultaneously detects DNA of Mycobacterium tuberculosis complex (MTBC) and resistance to rifampin (RIF) (i.e. mutation of the rpoB gene) in less than 2 hours. In comparison, standard cultures can take 2 to 6 weeks for MTBC to grow and conventional drug resistance tests can add 3 more weeks. The primers in the XpertMTB/RIF assay amplify a portion of the rpo $B$ gene containing the 81 base pair "core" region. The probes are able to differentiate between the conserved wild-type sequence and mutations in the core region that are associated with rifampicin resistance. WHO endorsed the gene Xpert MTB assay in detecting pulmonary TB and has a high sensitivity in smearnegative pulmonary TB. Recently, there has been increasing evidence that supports the use of this assay in clinical samples other than sputum from patients with Extra Pulmonary TB

\section{Aims and Objectives}

To study the role of CBNAAT in the rapid and early diagnosis of tuberculosis in both pulmonary and extra pulmonary specimens which in turn helps in the early diagnosis and treatment.

\section{Materials and Methods}

Participants were samples from both outpatients and inpatients attending the tertiary care centre. About 6638 samples have been taken from July 2017 to 2018 December .Out of which 1152 samples detected Mycobacterium tuberculosis. Out of this 1111 were sensitive to rifampicin and 41 were resistant to rifampicin. 5486 samples did not detect Mycobacterium tuberculosis. 41 cases where positive for HIV. 79 samples were extrapulmonary which included specimens from CSF, Pleural fluid\& Ascitic fluid.

\section{Results}

Out of 6638 samples collected 1152 samples were detected to have MTB, remaining 5486 did not detect MTB, out of this 1111 were sensitive to rifampicin while 41 samples were resistant to rifampicin.79 samples were extra pulmonary specimens .41 samples had HIV coinfection.

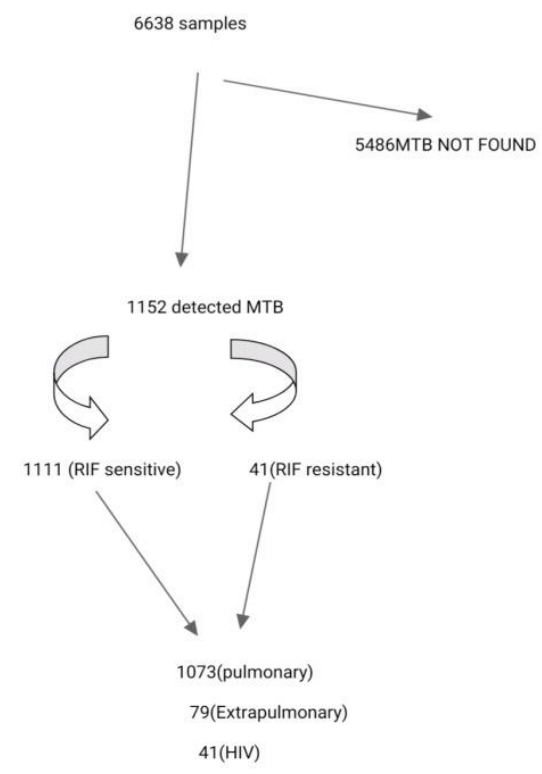

\begin{tabular}{|l|c|c|}
\hline $\begin{array}{l}1152 \text { (MTB detected } \\
\text { samples) }\end{array}$ & $\begin{array}{c}\text { Rifampicin } \\
\text { Sensitive }\end{array}$ & $\begin{array}{c}\text { Rifampicin } \\
\text { Resistant }\end{array}$ \\
\hline Pulmonary : 1073 & 1043 & 30 \\
\hline Extrapulmonary: 79 & 68 & 11 \\
\hline $\mathbf{1 1 5 2}$ & $\mathbf{1 1 1 1}$ & $\mathbf{4 1}$ \\
\hline
\end{tabular}

Out of this 1152 samples 1073 were pulmonary and 79 were extra pulmonary specimens .Among pulmonary specimens 1043 were sensitive to rifampicin while 30 were resistant to rifampicin and in 79 extrapulmonary specimens 68 were sensitive to rifampicin and 11 specimen were resistant to rifampicin. Most of the samples were pulmonary specimens(93\%), only around $7 \%$ are extrapulmonary specimens. HIV positive cases constitute around $3 \%$ of the total samples. $96 \%$ samples were sensitive to Rifampicin. $3.5 \%$ of samples were resistant to Rifampicin. For 
pulmonary specimens, the sensitivity and specificity of the MTB/RIF test were $96 \%$ and $100 \%$, respectively. For extrapulmonary specimens, the sensitivity and specificity were $68 \%$ and $100 \%$, respectively. In our study, 41 were Rifampicin-resistant and 1111 were Rifampicin sensitive samples were detected correctly by the CBNAAT assay. It has been known that for tuberculosis detection this MTB/RIF assay software requires a sample to have at least two positive probes with a change in the cycle threshold value (CT) of 2 cycles (3). This test has a short turnaround time and simultaneously detects M. tuberculosis and RIF resistance in less than 3 hours. Around $70 \%$ of the samples were from males and $30 \%$ were from females. In Age distribution most samples were above the age group of 15 years the youngest being of age 9 years and the oldest age being 75 years .Among the extra pulmonary specimens the maximum yield was from pleural fluid ,followed by CSF \& Ascitic fluid.

\section{Sensitivity and Specificity of Specimens}

\begin{tabular}{|l|c|c|}
\hline & $\begin{array}{c}\text { Pulmonary } \\
\text { Specimens }\end{array}$ & $\begin{array}{c}\text { Extra Pulmonary } \\
\text { Specimens }\end{array}$ \\
\hline Sensitivity & $96 \%$ & $68 \%$ \\
\hline Specificity & $100 \%$ & $100 \%$ \\
\hline Rifampicin Sensitivity & $90 \%$ & $82 \%$ \\
\hline Rifampicin Resistance & $36 \%$ & $25 \%$ \\
\hline
\end{tabular}

GENDER DISTRIBUTION:

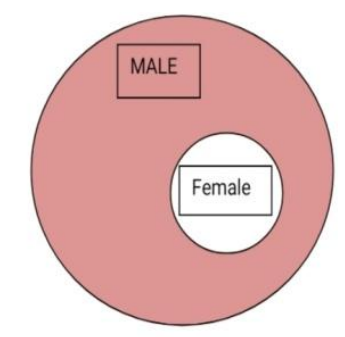

AGE DISTRIBUTION:

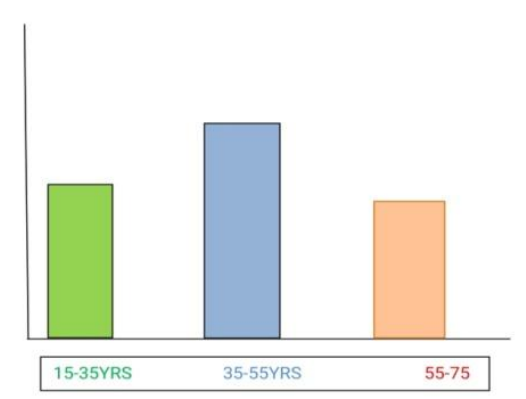

\section{Discussion}

In our study, the performance of the MTB/RIF assay with both pulmonary and extrapulmonary specimens obtained during the clinical routine were investigated. In all tuberculosis cases the sensitivity of the MTB/ RIF assay for pulmonary specimens were statistically higher than that for the extrapulmonary specimens. It could be because of high smear-negativity for the extrapulmonary specimens. Another study found that the MTB/RIF assay had a calculated limit of detection of $131 \mathrm{CFU} / \mathrm{ml}$ of sputum and was able to detect as few as $10 \mathrm{CFU} / \mathrm{ml}$ of sputum in $35 \%$ of samples (3). In general practice the MTB/RIF test is quite faster within 3 to 24 hours than the culture which require about 19 days. Among the infectious diseases Tuberculosis (TB) is one of the most dangerous threats to public health. As per the Global TB report 2017 the estimated incidence of $\mathrm{TB}$ in India was approximately 28,00,000 accounting for about a quarter of the world's TB cases .The National Strategic Plan (NSP) 20172025 for TB Elimination is a framework to provide guidance for the activities of National and State Governments, Civil Society Organizations, International Agencies, Research Institutions, Private Sector, and many others whose work is relevant to TB elimination in India The goal is to achieve a rapid decline in Burden of $\mathrm{TB}$, morbidity and mortality while working towards elimination of TB in India by 2025.GeneXpert MTB/RIF assay is a nucleic acid amplification (NAA) test which simultaneously detects DNA of Mycobacterium tuberculosis complex (MTBC) and resistance to rifampin (RIF) (i.e. mutation of the rpoB gene in less than 2 hours. In comparison, standard cultures can take 2 to 6 weeks for MTBC to grow and conventional drug resistance tests can add 3 more weeks. The primers in the XpertMTB/RIF assay amplify a portion of the $r p o B$ gene containing the 81 base pair "core" region. The probes are able to differentiate between the conserved wild-type sequence and mutations in the core region that are associated with rifampicin resistance. WHO 
endorsed the gene Xpert MTB assay in detecting pulmonary TB and has a high sensitivity in smearnegative pulmonary TB . Recently, there has been increasing evidence that supports the use of this assay in clinical samples other than sputum from patients with Extra Pulmonary TB. when compared to the gender distribution around $70 \%$ of the samples were from males ,30\% were from females, In Age distribution most specimens were from the age group of 15 years and above and the youngest being of age 9 years and the oldest age around 75 years of age. Among the extra pulmonary specimens the maximum yield were obtained from pleural fluid, followed by CSF \& Ascitic fluid.

\section{Conclusion}

From this study we have concluded that CBNAAT is a simple and cost effective method to detect MTB in pulmonary and extra pulmonary specimens. This assay has better sensitivity and specificity for the diagnosis of $\mathrm{TB}$ and detection of rifampin resistance in few hours. So this assay has a higher clinical application value in earlier diagnosis of both pulmonary and extra pulmonary TB which can be applied for the early diagnosis and treatment of the disease and to avoid injudicious use of Anti TB drugs and to reduce the mortality and morbidity from Tuberculosis.

\section{References}

1. Mani, C., N. Selvakumar, S. Narayanan, and P. R. Narayanan. 2001. Mutations in the rpoB gene of multidrug-resistant Mycobacterium tuberculosis clinical isolates from India. J. Clin. Microbiol. 39:2987-2990.

2. Makinen, J., H. J. Marttila, M. Marjamaki, M. K. Viljanen, and H. Soini. 2006. Comparison of two commercially available DNA line probe assays for detection of multidrug-resistant Mycobacterium tuberculosis. J. Clin. Microbiol. 44:350-352.
3. Helb, D., et al. 2010. Rapid detection of Mycobacterium tuberculosis and rifampin resistance by use of on-demand, nearpatient technology. J. Clin. Microbiol. 48:229-237.

4. Li et al., Journal of Medical Microbiology 2017;66:910-914

5. Evaluation of the GeneXpert MTB/RIF Assay for Rapid Diagnosis of Tuberculosis and Detection of Rifampin Resistance in Pulmonary and Extrapulmonary Specimens Arzu N. Zeka'1 Sezai Tasbakan, $^{2}$ and Cengiz Cavusoglu ${ }^{3}$

6. Marlowe, E. M., et al. 2011. Evaluation of the Cepheid Xpert MTB/RIF assay for direct detection of Mycobacterium tuberculosis complex in respiratory specimens. J. Clin. Microbiol. 49:16211623.

7. Harrisons, Principles of Internal Medicine

8. Revised National Tuberculosis Control Programme National Strategic Plan For Tuberculosis Elimination 2017-2025

9. Boehme, C. C., et al. 2010. Rapid molecular detection of tuberculosis and rifampin resistance. N. Engl. J. Med. 363:1005-1015. 